MATEC Web of Conferences 1, 01005 (2012)

DOI: $10.1051 /$ matecconf/20120101005

(c) Owned by the authors, published by EDP Sciences, 2012

\title{
Uncertainty Analysis in the Noise Parameters Estimation
}

\author{
W. Batko ${ }^{1}$, P. Pawlik ${ }^{1}$ \\ ${ }^{1}$ AGH University of Science and Technology, Cracow, Poland
}

\begin{abstract}
The new approach to the uncertainty estimation in modelling acoustic hazards by means of the interval arithmetic is presented in the paper.

In the case of the noise parameters estimation the selection of parameters specifying the acoustic wave propagation in an open space as well as parameters which are required in a form of average values - often constitutes a difficult problem. In such case, it is necessary to determine the variance and then, related strictly to it, the uncertainty of model parameters. The application of the interval arithmetic formalism allows to estimate the input data uncertainties without the necessity of the determination their probability distribution, which is required by other methods of uncertainty assessment.

A successive problem in the acoustic hazards estimation is a lack of the exact knowledge of the input parameters. In connection with the above, the analysis of the modelling uncertainty in dependence of inaccuracy of model parameters was performed. To achieve this aim the interval arithmetic formalism representing the value and its uncertainty in a form of an interval - was applied.

The proposed approach was illustrated by the example of the application the Dutch RMR SRM Method, recommended by the European Union Directive 2002/49/WE, in the railway noise modelling.
\end{abstract}

\section{Introduction}

The obligation of the realisation of acoustic maps is one of the requirements of the Directive 2002/49/WE [1], dealing with the guidelines for the estimation and maintenance of the environment noise level. Preparation of acoustic maps requires meeting several model and realisation recommendations [2]. In the transitory period an application of transitory methods or adopting own domestic methods is allowed, however under the condition of their adjusting for calculations of new noise estimation indices. There are four basic noise types: industrial, air, railway and road traffic. However, the transitory methods do not have strictly defined ways of uncertainty determinations, which is significant at such effects. The noise level modelling results depend on initial parameters, which are burdened with uncertainties (e.g. average vehicle speed, average number of vehicles). In addition, utilising the proposed computational methods requires a sound knowledge, among others, at selecting the input parameters of the model, which in the majority of cases can not be given as one number only.

The method of doing calculations on intervals, due to which the model parameters can be shown in a form of intervals representing their variability is presented in the hereby paper and in the previous ones [3,4]. The formalism of the interval arithmetic describes dependencies and operations on intervals. The application of this method was presented on the Dutch RMR SRM Method of the railway noise modelling (recommended by the European Directive) published in paper [5].

\section{Interval arithmetic formalism}

Operations on intervals had their beginning in the year 1950 , and in 60 -th they were named by Moore as interval arithmetic [6,7]. That time already it was applied for uncertainty estimations.

Intervals determine measuring and computational uncertainty - presented in this paper. All calculations in the noise modelling are done in the interval arithmetic. It is realised in such a way that at each computation stage, apart from the result, it is possible to determine its uncertainty.

Intervals are described as closed and limited sets of real numbers e.g.:

$\mathbf{x}=[\underline{x}, \bar{x}]=\{x \in R: \underline{x} \leq x \leq \bar{x}\}$

where $\underline{x}$ is the lower interval limit - infimum, $\bar{x}$ is the upper interval limit - supremum, $x$ - is the arbitrary number belonging to the interval. Interval numbers will be written in bold.

The basic mathematical operations are defined on the set of intervals: 
$\mathbf{x} \oslash \mathbf{y}=\{z=x \diamond y \quad: \quad x \in \mathbf{x} \quad y \in \mathbf{y}\}$

where $\diamond$ is one of the operators: addition, subtraction, multiplication or division. These operators, apart from division, are defined for arbitrary intervals. For the division it should be assumed that $0 \notin \mathrm{y}$.

Additions and subtractions of intervals is realised by operation on the interval endpoints, according to equations (3) and (4):

$$
\begin{aligned}
& \mathbf{x}+\mathbf{y}=[\underline{x}+\underline{y}, \bar{x}+\bar{y}] \\
& \mathbf{x}-\mathbf{y}=[\underline{x}-\bar{y}, \bar{x}-\underline{y}]
\end{aligned}
$$

The interval being the multiplication result is determined on the basis of the smallest and largest product of two endpoints of intervals $\mathrm{x}, \mathrm{y}$.

$$
\mathbf{x} \cdot \mathbf{y}=[\min (\underline{x} \underline{y}, \underline{x} \bar{y}, \bar{x} \underline{y}, \bar{x} \bar{y}), \max (\underline{x} \underline{y}, \underline{x} \bar{y}, \bar{x} \underline{y}, \bar{x} \bar{y})]
$$

The division operation is determined by means of the inverse:

$$
\frac{\mathbf{x}}{\mathbf{y}}=\mathbf{x} \cdot \frac{1}{\mathbf{y}}
$$

However, the interval inverse is given by (7):

$$
\frac{1}{\mathbf{y}}=\left[\frac{1}{\bar{y}}, \frac{1}{\underline{y}}\right] ; \text { when } \underline{y}>0 \text { or } \quad \bar{y}<0
$$

At the numerical realisation of interval operations there are constrains to present the results of a finite precision. Thus, the elementary functions are implemented by means of the Taylor series, since then the error caused by omitting expressions of a higher order is taken into account in the final result. For the monotonic function (e.g. exp, log), the result interval is given at the estimation of the rounding applied at elementary functions.

\section{Characteristics of the railway noise model}

In the case of modelling the railway noise the Dutch Method RMR SRM is the method recommended by the Directive 2002/49/WE. This method is reduced to the time-averaged sound level $L_{\text {Aeq }}$ determination. The first step at the modelling is the qualification of vehicles moving on the given railway track. The method differentiates 9 train categories. Then the value of the emission level is determined according to the dependence (8):

$$
E=10 \log \left(\sum_{c=1}^{y} 10^{E_{n r, c} / 10}+\sum_{c=1}^{y} 10^{E_{r, c} / 10}\right)
$$

where:

$E_{n r, c}-$ emission level for not braking trains belonging to the considered category,

$E_{r, c}-$ emission level for braking trains,

$c$ - train category,

$y \quad-$ total number of existing categories.

The emission level value for each category is determined according to $(9,10)$.

$$
\begin{aligned}
& E_{n r, c}=a_{c}+b_{c} \log v_{c}+10 \log Q_{c}+C_{b, c} \\
& E_{n r, c}=a_{r, c}+b_{r, c} \log v_{c}+10 \log Q_{r, c}+C_{b, c}
\end{aligned}
$$

where:

$Q_{c} \quad$ - average number of not braking trains belonging to the considered category,

$Q_{r, c}$ - average number of braking trains belonging to the considered category,

$v_{c} \quad$ - average speed of trains,

$a_{c}, b_{c}, a_{r, c}, b_{r, c}-$ standard values of emission level, depending on the category, quoted in paper [5],

$C_{b, c}$ - coefficient of correction depending on the category and type of rails, given in paper [5].

The equivalent sound level $L_{\text {Aeq }}$ for the railway noise is estimated from the dependence:

$$
L_{\text {Aeq }}=E_{S}+C_{\text {reflection }}-D_{\text {distance }}-D_{\text {air }}-D_{\text {soil }}-D_{\text {meteo }}
$$

where:

$C_{\text {reflection }}-$ correction for possible reflections from buildings or other vertical surfaces,

$D_{\text {distance }}$ - correction taking into account $r$ (distance of the receiving point from the source line),

$D_{\text {air }} \quad-$ correction taking into account a sound suppression in the air - depended on $r$.

$D_{\text {soil }} \quad$ - correction taking into account a sound absorption by the site; depended on $r, h_{w}$ (the receiving point height - in relation to the local height of the site), $h_{b s}$ (the rail head height in relation to the assessment surface) and on $B$ (soil coefficient),

$D_{\text {meteo }}-$ meteorological correction depended on $h$ and $h_{b s}$,

$E_{s} \quad-$ complex value of the emission level.

On account of the fact, that some input parameters are taken as average values $\left(v_{c}, Q_{c}, Q_{r, c}\right)$, it is necessary to estimate their variances and then take into consideration their uncertainties in modelling. In addition, parameters related to measurements of distances, heights or angles, which are performed with a defined accuracy and this accuracy can significantly influence the estimate of the considered noise levels. Parameters and their variability were written in the form of intervals, while all model calculations were performed in accordance with the interval arithmetic formalism. 


\section{Example of modelling by means of the interval arithmetic}

The authors applied the computational formalism of the interval arithmetic for the estimation of the influence of the input parameters uncertainty on the modelling results uncertainty. Input parameters $v_{c}, Q_{c}, r, h_{w}, h_{b s}$ were determined by means of interval numbers, representing the possible range of their variability - in the following way:

$\boldsymbol{v}_{\boldsymbol{c}}=\left[v_{c}-v_{c} \cdot k / 100, v_{c}+v_{c} \cdot k / 100\right]$

$Q_{c}=\left[Q_{c}-Q_{c} \cdot k / 100, Q_{c}+Q_{c} \cdot k / 100\right]$

$\boldsymbol{r}_{c}=\left[r_{c}-r_{c} \cdot k / 100, r_{c}+r_{c} \cdot k / 100\right]$

$\boldsymbol{h}_{w}=\left[h_{w}-h_{w} \cdot k / 100, h_{w}+h_{w} \cdot k / 100\right]$

$\boldsymbol{h}_{b s}=\left[\mathrm{h}_{b s}-\mathrm{h}_{b s} \cdot k / 100, \mathrm{~h}_{b s}+\mathrm{h}_{b s} \cdot k / 100\right]$

where: $\mathrm{k} \in\langle 0,15\rangle$ determines the uncertainty value in percentages. Due to the application of interval numbers the uncertainty is determined at each stage of model calculations.

Next, the simulation of the parameters uncertainty influence on the results of the equivalent sound levels for four cases was performed. All model calculations were carried out by means of the interval arithmetic formalism.

The figure below (Fig. 1) presents the variability range of the equivalent sound level in dependence on the input parameters uncertainty for 4 cases.

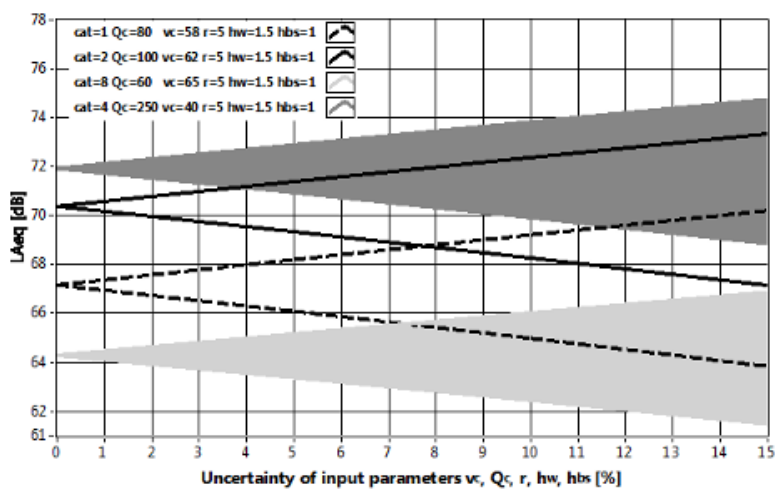

Fig. 1. Sound levels in a form of interval numbers in dependence on the input parameters uncertainty

As can be seen from the presented simulations the uncertainty of the input parameters has a significant influence on the estimation result. At $15 \%$ uncertainty of the model parameters the result uncertainty is \pm 2.5 $\mathrm{dB}$.

Table 1 presents the uncertainty of results expressed in percentages in dependence of the input parameters uncertainty, also expressed in percentages.
Table 1. Uncertainty of the estimated sound levels in dependence on the input parameters uncertainty $\mathrm{v}_{\mathrm{c}}, \mathrm{Q}_{\mathrm{c}}, \mathrm{r}, \mathrm{h}_{\mathrm{w}}$, $\mathrm{h}_{\mathrm{bs}}$.

\begin{tabular}{|c|c|c|c|}
\hline 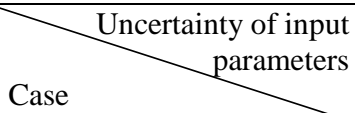 & $5[\%]$ & $10[\%]$ & $15[\%]$ \\
\hline 1 & $1.6[\%]$ & $3.2[\%]$ & $4.8[\%]$ \\
\hline 2 & $1.5[\%]$ & $2.9[\%]$ & $4.4[\%]$ \\
\hline 3 & $1.4[\%]$ & $2.8[\%]$ & $4.2[\%]$ \\
\hline 4 & $1.4[\%]$ & $2.7[\%]$ & $4.1[\%]$ \\
\hline
\end{tabular}

\section{Conclusions}

On account of the lack of the exactly defined methods for the determination uncertainties of acoustic maps, the formalism of the interval arithmetic was proposed. This approach does not require the knowledge on the probability distribution of model parameters, which enables its application in modelling the environment acoustic hazards.

The performed analyses indicate the essential influence of inaccuracy of input parameters on the result of the equivalent sound level modelling, which confirms the necessity of the uncertainty evaluation in these types of models.

The method of the uncertainty determination presented in this paper can be also applied for other noise models: road traffic, air and industrial noise.

\section{References}

1. Directive 2002/49/WE of the European Parliament and of the Council of 25 June 2002, relating to the assessment and management of environmental noise, Official Journal of the European Communities (2002)

2. Good Practice Guide for Strategic Noise Mapping and the Production of Associated Data on Noise Exposure. Version 2, European Commission Working Group Assessment of Exposure to Noise (2006)

3. W. Batko, P. Pawlik, Archives of Acoustics, vol. 35 no. 2, p. 278 (2010)

4. W. Batko, P. Pawlik, Archives of Acoustics, vol. 36 no. 3, p. 667 (2011)

5. Reken-en Meetvoorschrift Railverkeerslawaai '96, Ministerie Volkshuisveting, Ruimtelijke Ordening en Milieubeheer (1996)

6. R. E. Moore, Interval Arithmetic and Automatic Error Analysis in Digital Computing, Ph.D. Thesis, Stanford University (1962)

7. R. E. Moore, Interval Analysis, Prentice-Hall, Englewood Cliffs, NJ, USA (1966) 